\title{
Universiteit
}

Leiden

The Netherlands

\section{Phase behavior in thin films of cylinder-forming ABA block copolymers: mesoscale modeling}

Horvat, A.; Lyakhova, K.S.; Sevink, G.J.A.; Zvelindovsky, A.V.; Magerle, R.

\section{Citation}

Horvat, A., Lyakhova, K. S., Sevink, G. J. A., Zvelindovsky, A. V., \& Magerle, R. (2004). Phase behavior in thin films of cylinder-forming ABA block copolymers: mesoscale modeling. Journal Of Chemical Physics, 120(2), 1117-1126. doi:10.1063/1.1627325

Version: $\quad$ Not Applicable (or Unknown)

License: $\quad$ Leiden University Non-exclusive license

Downloaded from: https://hdl.handle.net/1887/64327

Note: To cite this publication please use the final published version (if applicable). 


\section{Phase behavior in thin films of cylinder-forming ABA block copolymers: Mesoscale modeling}

A. Horvat, K. S. Lyakhova, G. J. A. Sevink, A. V. Zvelindovsky, and R. Magerle

Citation: The Journal of Chemical Physics 120, 1117 (2004); doi: 10.1063/1.1627325

View online: https://doi.org/10.1063/1.1627325

View Table of Contents: http://aip.scitation.org/toc/jcp/120/2

Published by the American Institute of Physics

\section{Articles you may be interested in}

Phase behavior in thin films of cylinder-forming ABA block copolymers: Experiments

The Journal of Chemical Physics 120, 1105 (2004); 10.1063/1.1627324

Block Copolymers-Designer Soft Materials

Physics Today 52, 32 (1999); 10.1063/1.882522

Thin films of block copolymer

The Journal of Chemical Physics 106, 7781 (1997); 10.1063/1.473778

Equilibrium behavior of symmetric ABA triblock copolymer melts

The Journal of Chemical Physics 111, 7139 (1999); 10.1063/1.480006

Asymmetric block copolymers confined in a thin film

The Journal of Chemical Physics 112, 2452 (2000); 10.1063/1.480811

Fluctuation effects in the theory of microphase separation in block copolymers

The Journal of Chemical Physics 87, 697 (1987); 10.1063/1.453566

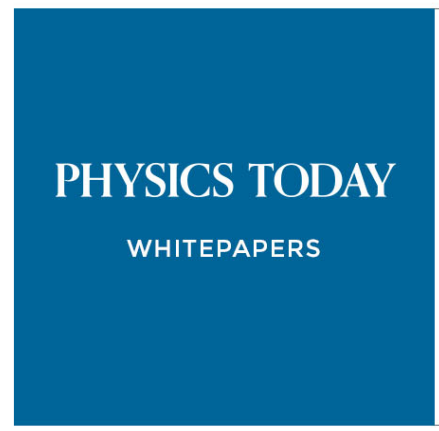




\title{
Phase behavior in thin films of cylinder-forming $A B A$ block copolymers: Mesoscale modeling
}

\author{
A. Horvat \\ Physikalische Chemie II, Universität Bayreuth, D-95440 Bayreuth, Germany \\ K. S. Lyakhova, G. J. A. Sevink, and A. V. Zvelindovsky \\ Leiden Institute of Chemistry, Leiden University, P.O. Box 9502, 2300 RA Leiden, The Netherlands \\ R. Magerle ${ }^{\mathrm{a})}$ \\ Physikalische Chemie II, Universität Bayreuth, D-95440 Bayreuth, Germany
}

(Received 24 June 2003; accepted 25 September 2003)

\begin{abstract}
The phase behavior of cylinder-forming $A B A$ block copolymers in thin films is modeled in detail using dynamic density functional theory and compared with recent experiments on polystyrene-block-polybutadiene-block-polystyrene triblock copolymers. Deviations from the bulk structure, such as wetting layer, perforated lamella, and lamella, are identified as surface reconstructions. Their stability regions are determined by an interplay between surface fields and confinement effects. Our results give evidence for a general mechanism governing the phase behavior in thin films of modulated phases. (C) 2004 American Institute of Physics.
\end{abstract}

[DOI: $10.1063 / 1.1627325]$

\section{INTRODUCTION}

Block copolymers self-assemble into ordered structures with characteristic lengths determined by the molecular size, which is in the $10-100 \mathrm{~nm}$ range. ${ }^{1,2}$ This property has attracted much interest in the area of soft condensed matter physics and nanotechnology. There is large interest to understand, predict, and control structure formation in this class of ordered polymeric fluids.

In bulk, the block copolymer microdomain structure is determined mainly by the molecular architecture and the interaction between the different components (blocks). At the air-polymer interface and the film-substrate interface additional driving forces for structure formation exist. Typically, one component has a lower interfacial energy than the other. This causes a preferential attraction of one type of block to the interface (or surface), which can result either in an alignment of the bulk structure at the interface ${ }^{3-5}$ and/or a deviation of the microdomain structure from the bulk. These deviations in the vicinity of the interface have been shown to be analogous to surface reconstructions of crystal surfaces. ${ }^{6}$

In thin films, additional constraints play an important role. Here, the microdomain structure has to adjust to two interfaces and a certain film thickness, which can be a noninteger multiple of the microdomain spacing in the bulk. Both constraints together cause a complex and interesting phase behavior.

Since the seminal work of Anastasiadis et al., ${ }^{3}$ the behavior of lamellae forming block copolymers in thin films has been studied in detail and two major effects have been identified (for reviews, see Refs. 7-9). The preferential attraction of one type of block to the surface (the surface field)

\footnotetext{
a) Author to whom all correspondence should be addressed. Electronic mail: robert.magerle@uni-bayreuth.de
}

causes the lamella to align parallel to interfaces and the film forms islands or holes where the film thickness is a (half) integer multiple of the lamella spacing in the bulk. In cases where the film thickness is not compatible with the natural bulk domain spacing or when the film/air and the film/ substrate interface is not selective, lamellae can orient perpendicular to the interfaces. ${ }^{4,5}$

The behavior of cylinder forming systems is more complex and less understood. Here, the natural hexagonal packing of cylinders cannot be retained close to the planar interface, which, regardless of its orientation, always breaks the symmetry of the bulk structure. As a result, besides cylinders oriented parallel and perpendicular to the surface, ${ }^{10-13}$ a variety of deviations from the bulk structure have been observed near surfaces and in thin films, such as a disordered phase, ${ }^{10}$ a wetting layer, ${ }^{14}$ spherical microdomains, ${ }^{15}$ a perforated lamella, ${ }^{15}$ as well as more complicated hybrid structures such as cylinders with necks, ${ }^{16}$ a perforated lamella with spheres, ${ }^{17}$ and an inverted phase. ${ }^{18}$

Various theories have been used to describe this behavior. ${ }^{19-26}$ A brief summary of experimental and simulation results is given in Ref. 24. With dynamic density functional theory (DDFT), a dynamic variant of self-consistent field theory, Huinink et al. ${ }^{21,22}$ have calculated a phase diagram for thin films of cylinder forming diblock copolymers. They found that noncylindrical structures are stabilized by the surface field in the vicinity of surfaces and in thin films. With increasing strength of the surface field a sequence of phase transitions was predicted: from a wetting layer, to cylinders oriented perpendicular to the surface, to cylinders oriented parallel to the surface, to a hexagonally perforated lamella, and to a lamella.

Theoretical and experimental results agree qualitatively only in part. From the experimental point of view, only single deviations from the bulk structure and no phase dia- 


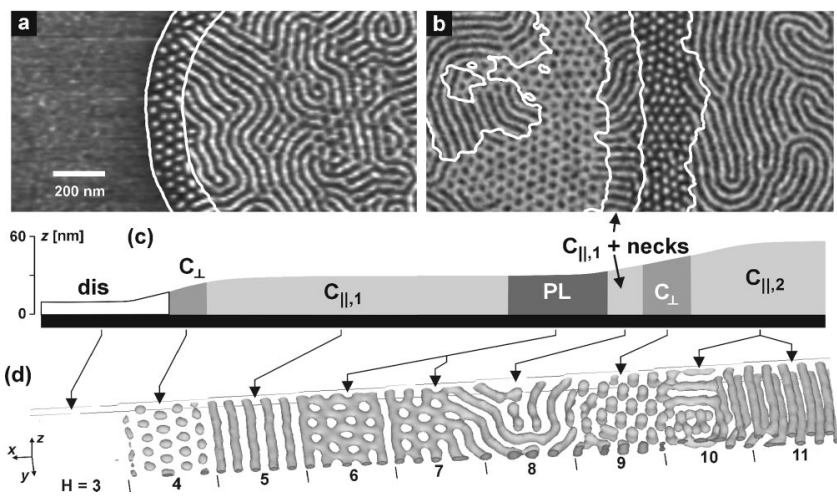

FIG. 1. (a), (b) TappingMode ${ }^{\mathrm{TM}}$ scanning force microscopy phase images of thin polystyrene-block-polybutadiene-block-polystyrene (SBS) films on silicon substrates after annealing in chloroform vapor. The surface is covered with a homogeneous $\approx 10$-nm-thick PB layer. Bright (dark) corresponds to PS (PB) microdomains below this top PB layer. Contour lines calculated from the corresponding height images are superimposed. (c) Schematic height profile of the phase images shown in (a) and (b). (d) Simulation of an $A_{3} B_{12} A_{3}$ block copolymer film in one large simulation box with from left to right increasing film thickness $H(x), \epsilon_{A B}=6.5$, and $\epsilon_{M}=6.0$. The isodensity surface $\rho_{A}=0.5$ is shown. [Reprinted from: A. Knoll et al., Phys. Rev. Lett. 89, 035501 (2002); (C2002 American Physical Society.]

grams have been reported. Therefore it remains unclear which of the reported phenomena are specific to the particular block copolymer and/or route of film preparation and which are general behavior. From the modeling point of view, no model predicts all experimentally observed phases. In particular, a detailed and quantitative comparison between modeling and experimental results is missing. The underlying fundamentals remain unclear.

Recently, Knoll et al. ${ }^{27}$ have measured the phase diagram for thin films of concentrated solutions of a polystyrene-block-polybutadiene-block-polystyrene (SBS) triblock copolymer in chloroform as function of film thickness and polymer concentration. In this communication ${ }^{27}$ we have presented matching computer simulations of thin films of $A B A$ triblock copolymers which model in stunning detail the experimentally observed phase behavior. As an example, Fig. 1 shows a comparison of the experimental results of Knoll et al. $^{27}$ and our simulations of a corresponding $A_{3} B_{12} A_{3}$ triblock copolymer film, where the interfaces preferentially attract the $B$ block. In Fig. 1 the film thickness increases from left to right and a rich variety of structures is observed. With increasing film thickness, both experiments and calculations show the same sequence of thin film phases: a disordered film (dis) for the smallest thickness, $A$ spheres or very short upright $A$ cylinders $\left(\mathrm{C}_{\perp}\right), A$ cylinders oriented parallel to the film plane $\left(\mathrm{C}_{\|, 1}\right)$, a perforated $A$ lamella (PL), parallel oriented $A$ cylinders with an elongated cross section and necks, perpendicular oriented $A$ cylinders $\left(\mathrm{C}_{\perp}\right)$, and finally two layers of parallel oriented $A$ cylinders $\left(C_{\|, 2}\right)$. The phase transitions occur at well-defined film thickness as can be seen from the white contour lines that represent points of equal film thickness [Figs. 1(a) and 1(b)].

As measured three-dimensional volume images of a thin film's microdomain structure are rather rare, ${ }^{16,17,28,29}$ DDFT simulations as shown in Fig. 1(d) facilitate us to interpret the experimentally easy observed surface structures in terms of the volume structure of the films. Furthermore, compared to the experiments ${ }^{27,30}$ and previous simulations on diblock copolymers, ${ }^{21,22}$ our simulations cover a much larger parameter space. Only this enabled us to distinguish between the different physical phenomena governing the phase behavoir in thin films. The phenomena and their interplay can be summarized in the following way: (1) The surface field can either orient the bulk structure or it can stabilize deviations from bulk structures, such as wetting layer, perforated lamella, and lamella, which we identified as surface reconstructions. ${ }^{6}$ (2) The film thickness is modulating the stability regions of the different phases via interference of surface fields and confinement effects.

The aim of this paper is to give a detailed report of our simulation results. The experimental part is reported in the preceeding companion article. ${ }^{30}$ First, we report briefly the phase behavior of an $A_{3} B_{12} A_{3}$ melt in the bulk. Then we focus on the phase behavior of cylinders forming systems in thin films. The basic types of surface structures and surface reconstructions are introduced and the underlying physics is explained. Finally, we compare our results with the corresponding experiments of Knoll et al. ${ }^{30}$ and the phase behavior of other cylinder and lamella forming block copolymers.

\section{METHOD}

We have modeled the phase behavior in thin films with mean-field DDFT, which was developed by Fraaije et al. ${ }^{31-33}$ for mesoscale modeling the phase separation and ordering processes of multicomponent polymeric systems. For our simulations we used the standard MESODYN code. ${ }^{34}$

As a molecular model an ideal Gaussian chain is used. In this "spring and beads" model, springs mimic the stretching behavior of a chain fragment and different kinds of beads correspond to different components in the block copolymer. All nonideal interactions are included via a mean field and the strength of interaction between different components is characterized by the interaction parameter $\epsilon_{A B}$, which we express in units of $\mathrm{kJ} / \mathrm{mol}$. This parameter can be related to the conventional Flory-Huggins parameter $\chi$ (see Sec. III A). Interfaces are treated as hard walls, with the flux perpendicular to the interface and the density at the interface kept equal to zero. ${ }^{33}$ The interaction between components and interfaces is characterized by corresponding mean field interaction parameters $\epsilon_{A M}$ and $\epsilon_{B M}$. As only the difference between the interaction parameters is relevant for structure formation, ${ }^{33}$ we used an effective interface-polymer interaction parameter $\epsilon_{M}=\epsilon_{A M}-\epsilon_{B M}$, which characterizes the strength of the surface field. A positive $\epsilon_{I J}$ parameter corresponds to a repulsion between the components $I$ and $J$. The dynamics of the component densities $\rho_{I}(\mathbf{r}, t)$, with $I=A, B$, is described by a set of functional Langevin equations. These are diffusion equations of the component densities which take into account the noise in the system. Driving forces for diffusion are local gradients of chemical potentials $\mu_{I}$ $=\delta F\left[\left\{\rho_{I}\right\}\right] / \delta \rho_{I}$. The Langevin diffusion equations are solved numerically with homogeneous initial conditions. As MESODYN is based on the same type of free energy functional as self-consistent field theory (SCFT), ${ }^{35}$ it is expected to approach on long time scales the same solutions as SCFT does 
by searching for the absolute minimum of the free energy. With MESODYN, however, structure formation proceeds via local gradients of chemical potentials that are intrinsic to the system. In this way, long lived transition states can also be visited in a simulation run. This ambiguity, however, is shared with the experiments, where the specimen is also quenched after a finite annealing time. ${ }^{30}$

The simulations were done on a cubic grid of dimensions $X \times Y \times(H+1)$, with the interface positioned at $z=0$. Due to the periodicity of boundary conditions, the system is confined between two interfaces separated by $H$ grid points. The triblock copolymer is modeled as a melt of $A_{3} B_{12} A_{3}$ chains, which can be seen as two connected $A_{3} B_{6}$ chains. The architecture of the $A_{3} B_{12} A_{3}$ chain enters specifically in the calculation of the density fields from the external potentials and in the partition function, respectively, the free energy. ${ }^{31-33}$ For our simulations we partly relied on previous results (Refs. 21 and 22). Apart from the chain architecture all simulation parameters are the same as for the $A_{3} B_{6}$ diblock system studied in Refs. 21 and 22, with an exception for the interaction parameter $\epsilon_{A B}$. In addition, we have varied this interaction parameter in a range where the $A_{3} B_{12} A_{3}$ system forms cylinders in the bulk. Doing so we can also study the influence of the molecular architecture on the observered phenomena by comparing our results on $A_{3} B_{12} A_{3}$ triblock copolymer with the behavior of the corresponding $A_{3} B_{6}$ diblock copolymer. At the same time, this study allowed us to determine the value of the interaction parameter $\epsilon_{A B}$ that best matches the experimental situation. As in Refs. 21 and 22, we followed the temporal evolution in the system until significant changes of the free energy, the order parameter, and the microdomain structure no longer occurred.

\section{RESULTS}

\section{A. Bulk structure}

As a first step, we parametrized the system studied experimentally by Knoll et al. ${ }^{27,30}$ For this, we investigated the phase behavior in the bulk and determine the range of $\epsilon_{A B}$ where the system forms cylinders. In Fig. 2 the phase diagrams of a melt of $A B A$ triblock copolymers with $A$-volume fraction $f_{A}=1 / 3$ are shown, which were calculated with DDFT and SCFT. ${ }^{36}$ The Flory-Huggins parameter $\chi$ and $\epsilon_{A B}$ are related through $\chi=\left(\nu^{-1} / 2 k T\right)\left[2 \epsilon_{A B}-\epsilon_{A A}-\epsilon_{B B}\right]$ [Eq. (32) in Ref. 32]. In our case, with $\nu=1, \epsilon_{A A}=\epsilon_{B B}=0, N$ $=18$, and $T=413 \mathrm{~K}, \chi N \approx \epsilon_{A B} \times 5.43 \mathrm{~mol} / \mathrm{kJ}$.

The phase separation process was simulated with DDFT in a cubic box with $32 \times 32 \times 32$ grid points and periodic boundary conditions. The calculations were started with a homogeneous melt. During the simulation run we observe similar processes as previously reported. ${ }^{31}$ First the segregation of the $A$ and $B$ blocks into interconnected domains takes place. The separation process continues with the break-up of an initially connected network of different domains into well-defined structures. Microdomains with different orientations form simultaneously during the phase separation process, which leads to a very defective structure. The last and slowest process is the long-range ordering of the microdomain structure, which proceeds via annealing of defects.

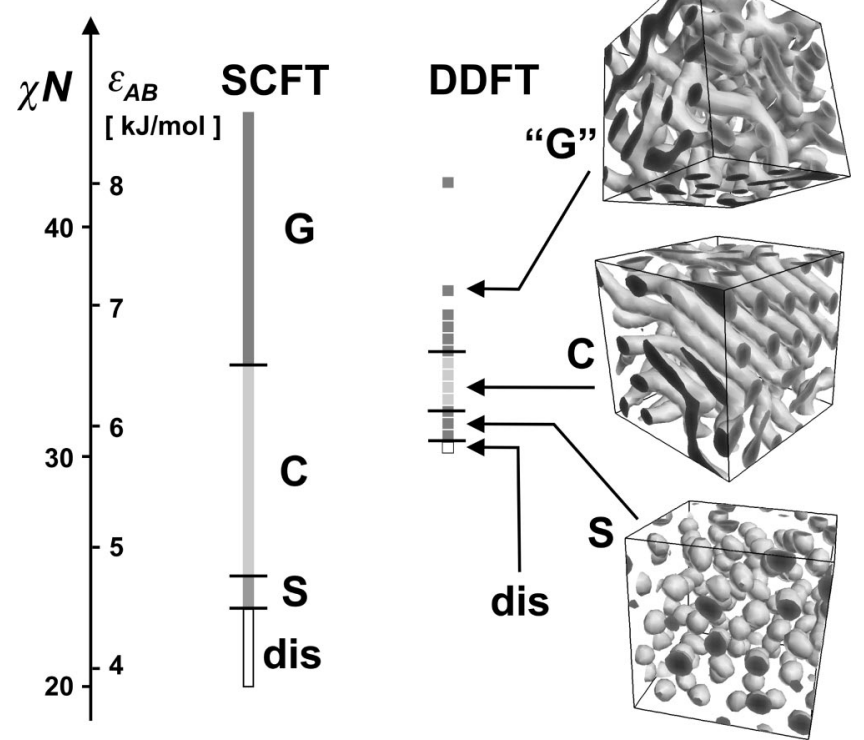

FIG. 2. Bulk phase diagrams for $A B A$ triblock copolymer melts with $f_{A}$ $=1 / 3$ as a function of interaction parameters $\chi N$ and $\epsilon_{A B}$. Results of Matsen (Ref. 30) obtained with SCFT are compared with our results calculated with DDFT for an $A_{3} B_{12} A_{3}$ melt in $32 \times 32 \times 32$ large simulation boxes. Phases are labeled as G (gyroid), "G" (gyroid-like), C (cylinders), S (spheres), and "dis" (disordered).

Our calculations were done until 4000 time steps, where the long-range ordering process is still not completed. Nevertheless, the result is sufficient to characterize the formed microdomain structure. For $\epsilon_{A B} \leqslant 5.75$ no phase separation occurs. The $A$ density $\rho_{A}$ is spatially inhomogeneous with a mean value (averaged over all grid points) of 0.33 and standard deviation of 0.03 . The mean value of 0.33 corresponds to the volume fraction $f_{A}$ of the $A$ component. As the interaction parameter $\epsilon_{A B}$ increases, $A$-rich domains of spherical shape (S) form in a $B$-rich matrix for $5.8 \leqslant \epsilon_{A B} \leqslant 6.0$. In the range $6.1 \leqslant \epsilon_{A B} \leqslant 6.5$, well-separated cylindrical $A$ microdomains (C) embedded in the $B$ matrix were observed. For $\epsilon_{A B}$ $\geqslant 6.6$, we observe an $A$-rich network of microdomains embedded in a $B$-rich matrix. Because of the large amount of three-fold connections we relate this structure to a defective gyroid phase. We have also done simulations in smaller simulation boxes, $16 \times 16 \times 16$ in size, and obtained similar results but with better ordered structures.

For $\epsilon_{A B}=6.5$ we determined the distance between cylinders in the bulk. For this purpose we did a simulation in a $64 \times 64 \times 1$ large box, analogous to Huinink et al. ${ }^{21}$ Here, due to the periodic boundary conditions, the cylinders orient perpendicular to the $64 \times 64$ plane and show up as hexagonally packed dots. The distance between next-nearest cylinders was determined to be $a_{0}=6.9 \pm 0.5$ grid units.

Our results are similar to those obtained with SCFT. With increasing interaction parameter both methods predict transitions from a disordered state to spheres, then cylinders, and a gyroid phase. We observe the transition from the disordered state to spheres at a higher value of $\epsilon_{A B}$ than predicted by Matsen et al. ${ }^{36}$ The discrepancy could be due to the relatively small size of the chain and the nonlocality of the nonideal interactions. ${ }^{32}$ The phase boundary between the cyl- 
a)

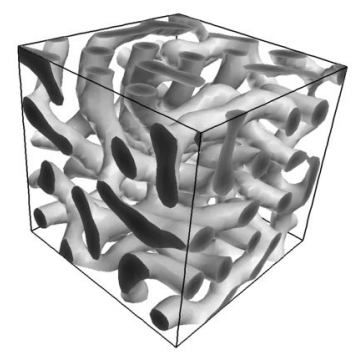

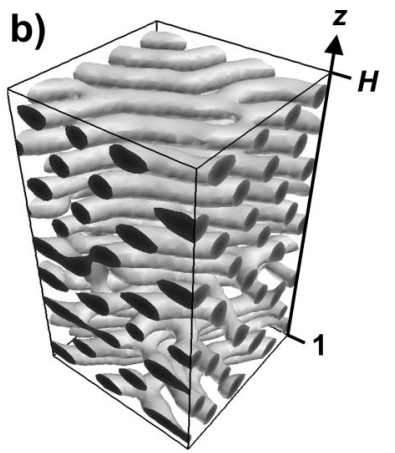

FIG. 3. Effect of the surface on the long range ordering process. Simulations for a cylinder forming system with interaction parameter $\epsilon_{A B}=6.5$ after 4000 time steps. (a) In the bulk, in a $32 \times 32 \times 32$ large simulation box. (b) In a confined film, where $X=Y=32$ and $H=54$, surfaces at $z=0$ and 55, and the effective surface-polymer interaction parameter $\epsilon_{M}=6$.

inder and gyroid phase is the same in both simulation results and this region of the phase diagram is of particular interest of the present study. An obvious difference is the presence of defects in the microdomain structures simulated with DDFT, which Matsen's SCFT does not take into account. If defects cost very little energy a rather high density of defects might be thermally excited in the system.

\section{B. Surface reconstructions}

We now turn to the question of what happens when interfaces are added to the system. On varying the film thickness, $H$, and the strength of the surface field, $\epsilon_{M}$, we observe a complex phase behavior. The presence of interfaces has several effects. One is a speed-up of the long range order formation. In Fig. 3 two systems with different boundary conditions and otherwise identical parameters are compared: an $A_{3} B_{12} A_{3}$ melt with $\epsilon_{A B}=6.5$ in the bulk [Fig. 3(a)] and in a film with $H=54$ [Fig. 3(b)]. The surface field was chosen to be $\epsilon_{M}=6$. In both systems, the simulation time was 4000 time steps and both show cylinders. In the film, the temporal evolution of structure formation is similar to that of a bulk system. In addition, however, the cylinders start to align at the interfaces and the alignment propagates from the surface into the film. This causes the cylinders in the film to orient parallel to the surface and to pack in a neat hexagonal array [Fig. 3(b)]. In the bulk, however, the microdomain structure is still very defective [Fig. 3(a)]. Although the simulation box of the film system is larger than that of the bulk simulation it shows a higher degree of long-range order.

The most intriguing effect of the presence of interfaces are deviations from the bulk microdomain structure in the vicinity of the interface. This effect is called surface reconstruction and it is best seen at large film thicknesses, for instance at $H=54$ (Fig. 4). In such films the interfaces are separated by approximately nine layers of cylinders and in the vicinity of one interface the influence of the other one is negligible. In the middle of the film, in most cases the microdomain structure remains hexagonally ordered cylinders aligned parallel to the film plane. Depending on the strength of the surface field, considerable rearrangements of microdomains near the interfaces, i.e., surface reconstructions, occur. For $\epsilon_{M}<2$, the $A$ component is preferentially attracted to the interface and a wetting layer (W) is formed. When $\epsilon_{M}$ increases, cylinders oriented perpendicular to the surface are stabilized for $\epsilon_{M} \approx 3$. As $\epsilon_{M}$ is further increased, the $A$ component is weakly repelled from the interface and cylinders orient parallel to the surface in the range $\epsilon_{M} \approx 4-9$. For larger $\epsilon_{M}$, surface reconstructions with noncylindrical microdomains are induced: first, at $\epsilon_{M} \approx 10$, a transition to a perforated lamellae (PL) occurs in the layer next to the surface which transforms to a lamellae (L) at $\epsilon_{M} \approx 25$.

For the surface structures shown in Fig. 4 we examined the density distribution of each component. In Fig. 5(a) the $(x, y)$ plane averaged $A$ density $\left\langle\rho_{A}\right\rangle_{x, y}$ is plotted as function of the distance $z$. For all three $\epsilon_{M}$ values $(5,10$, and 30), a modulation is observed, which corresponds to a layered microdomain structure oriented parallel to the interface. In all three of the displayed cases the $B$ component is attracted to the interface. This causes a depletion of the $A$ component at the interface and an increase of $\rho_{A}$ in the middle of the first $A$ microdomain next to the interface. The effect increases with increasing surface field $\epsilon_{M}$ and it is accompanied with formation of different surface reconstructions. At $z=3$, ap-

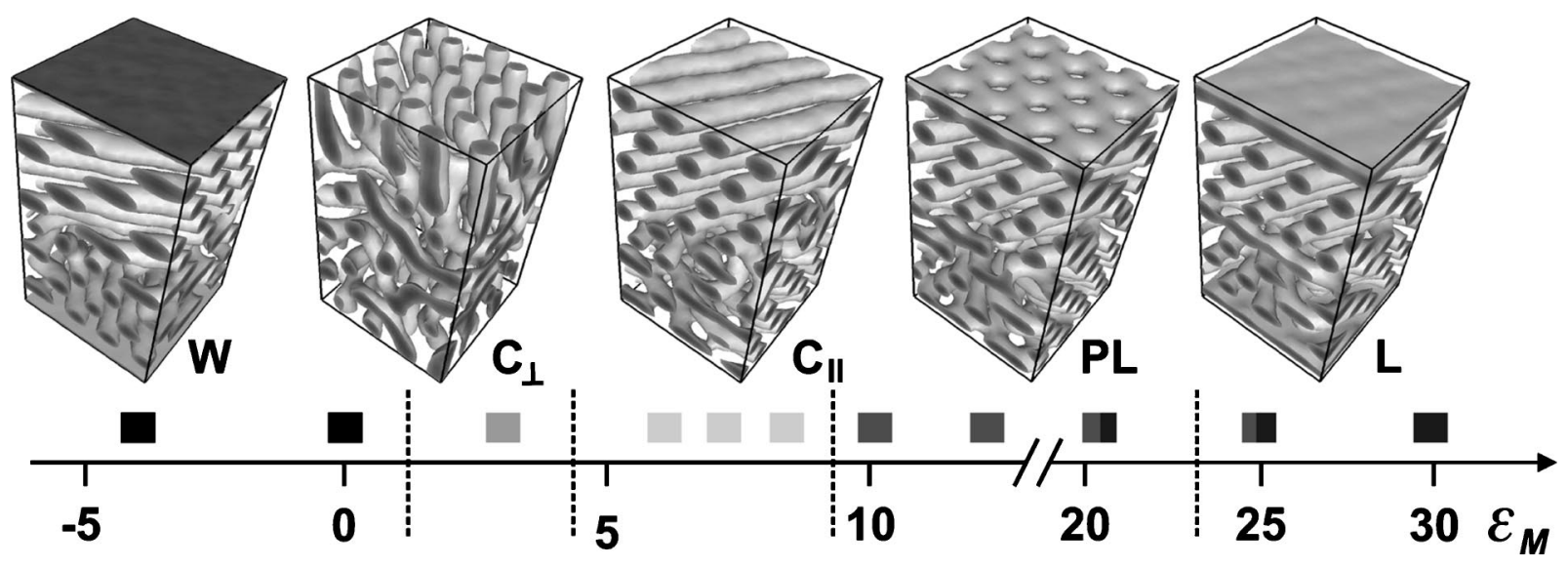

FIG. 4. Effect of the strength of the surface field $\epsilon_{M}$ on microdomain structures and surface reconstructions. Simulation results for an $A_{3} B_{12} A_{3}$ melt ( $\epsilon_{A B}$ $=6.5)$ in a rather thick film $(H=54)$ with surfaces at $z=0$ and 55 at $\epsilon_{M}=-4,3,7,12,30$. Isodensity surfaces $\left(\rho_{A}=0.45\right)$ are shown for typical structures. Gray boxes indicate $\epsilon_{M}$ values where simulations have been done. 

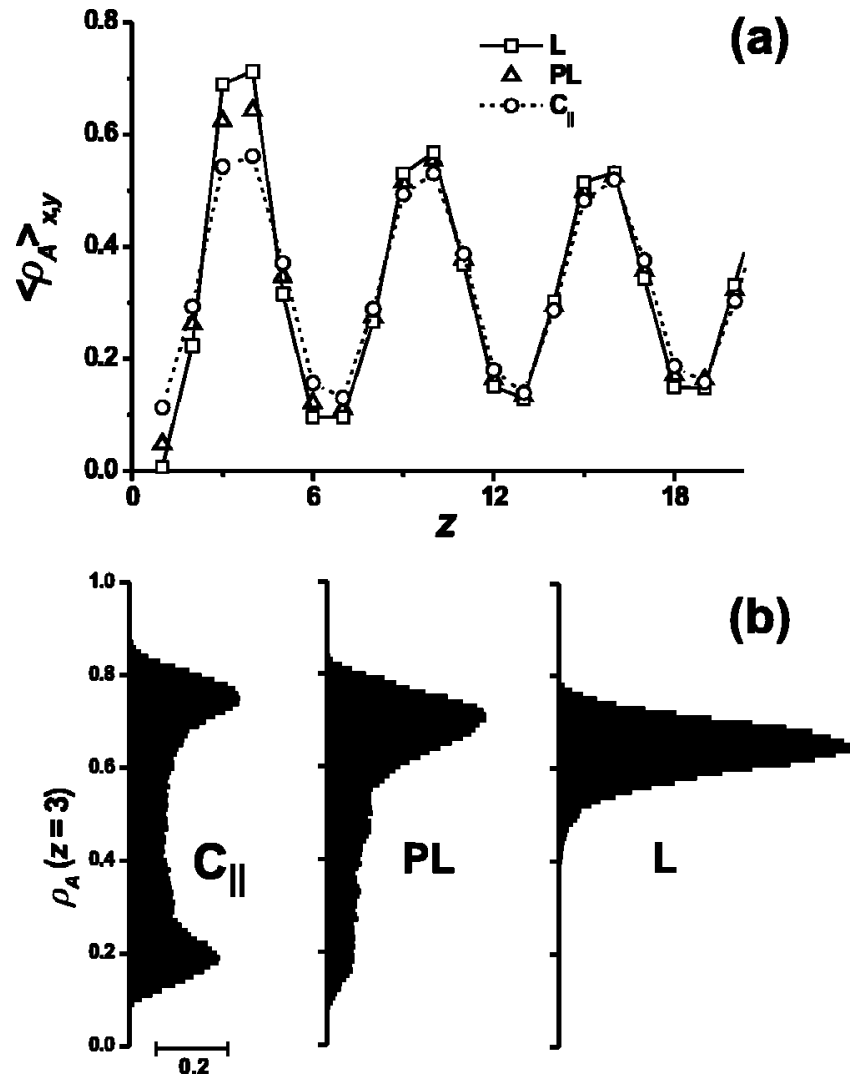

FIG. 5. Effect of the strength of the surface field on the distribution of $A$ density. (a) The laterally averaged $A$ density $\left\langle\rho_{A}\right\rangle_{x, y}$ as function of the distance $z$ from the surface (depth profiles) for different surface fields and surface reconstructions: $(\bigcirc)$ parallel cylinders, $\epsilon_{M}=5 ;(\triangle)$ perforated lamellae, $\epsilon_{M}=10$; $(\square)$ lamellae, $\epsilon_{M}=25$. (b) Histograms of the lateral averaged $A$ density $\left\langle\rho_{A}\right\rangle_{x, y}$ at $z=3$, approximately in the middle of the first $A$-rich microdomain next to the surface, for the surface fields shown in (a). proximately in the middle of the first $A$-rich microdomain next to the interface, $\rho_{A}$ increases with increasing surface field $\epsilon_{M}$ from $\rho_{A}=0.55$ for cylinders $\left(\epsilon_{M}=5\right)$, to $\rho_{A}=0.62$ for the perforated lamella $\left(\epsilon_{M}=10\right)$, and 0.70 for the lamella surface reconstruction $\left(\epsilon_{M}=30\right)$. In Fig. 5(b), the lateral distributions of the $A$ density at $z=3$ are plotted as histograms for the same values of the surface field as in Fig. 5(a). Results for $\epsilon_{M}=5$ show a broad density spectrum with two peaks, which correspond to the presence of two microphase separated components: $A$-rich cylinders and the $B$ matrix. For $\epsilon_{M}=12$, the distribution is still broad and $A$ is the majority component in this layer, the isodensity surface is a perforated lamella. For $\epsilon_{M}=30$, the density distribution shows one narrow peak, as expected for a lamella. These results indicate that with increasing surface field the density variations parallel to the interface are suppressed in the vicinity of the interface. In these structures the averaged mean curvature is gradually decreased in order to adopt to the planar symmetry of the interface.

\section{One microdomain thick films}

We now turn to the effect of the film thickness $H$. In thinner films two additional factors influence the microdomain structure in the film: the interference of the two surface fields (of the bottom and the top interface) and the commensurability of the natural domain spacing with the film thickness. First, we present the interference effect of surface fields for $H=6$, which corresponds to one layer of cylinders (Fig. 6). For this thickness we observe similar structures as in thick films $(H=54)$ in the vicinity of the interface. For $H=6$, however, the strength of surface field needed to form noncylindrical microdomains is strongly reduced. We ob-

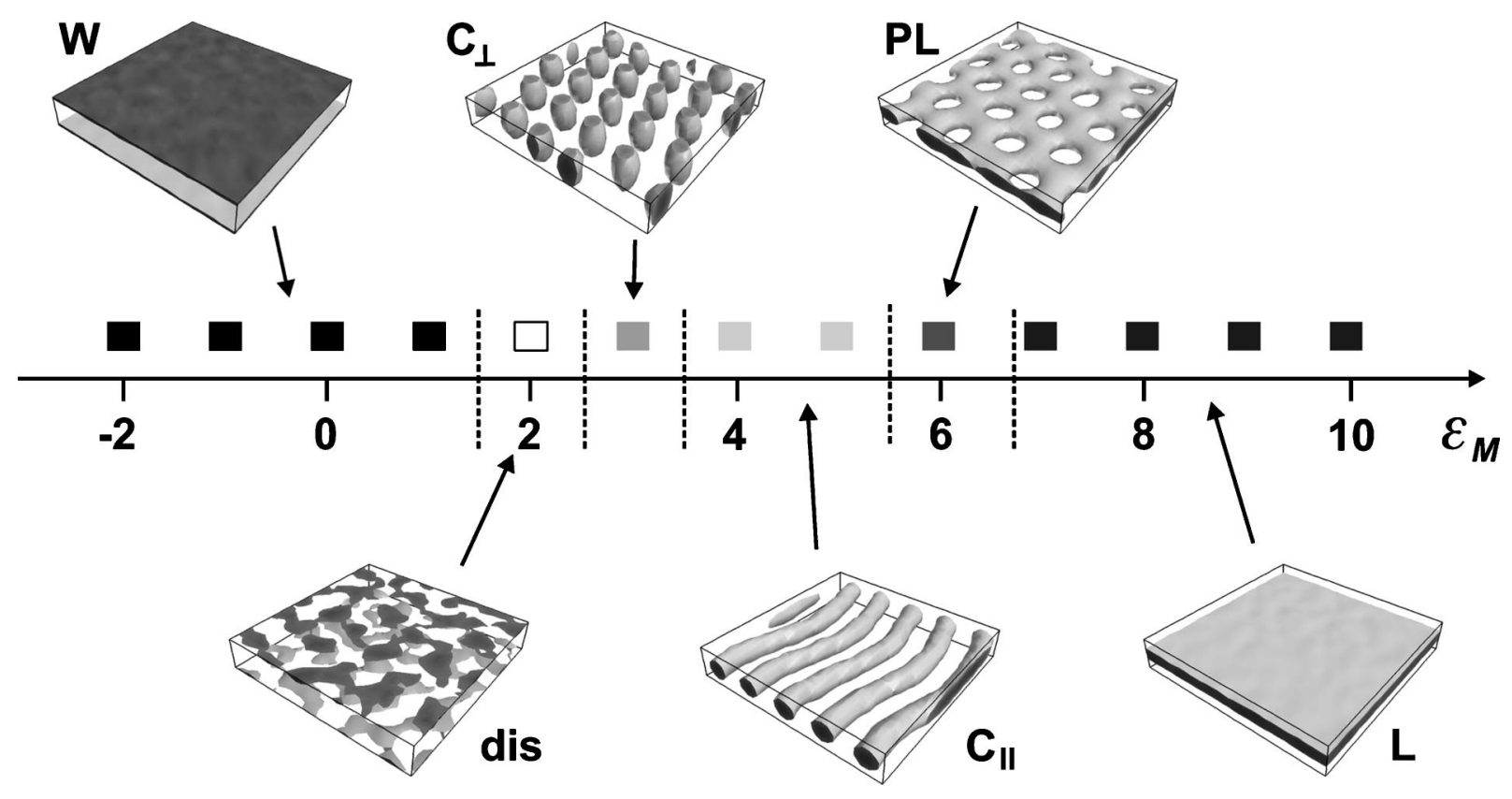

FIG. 6. Simulation results for a cylinder forming $A_{3} B_{12} A_{3}$ melt $\left(\epsilon_{A B}=6.5\right)$ in thin films $(H=6)$ with different strength of the surface field $\epsilon_{M}$. Isodensity profiles $(\rho=0.45)$ for typical structures are shown. Gray boxes indicate parameters were simulations have been done. 


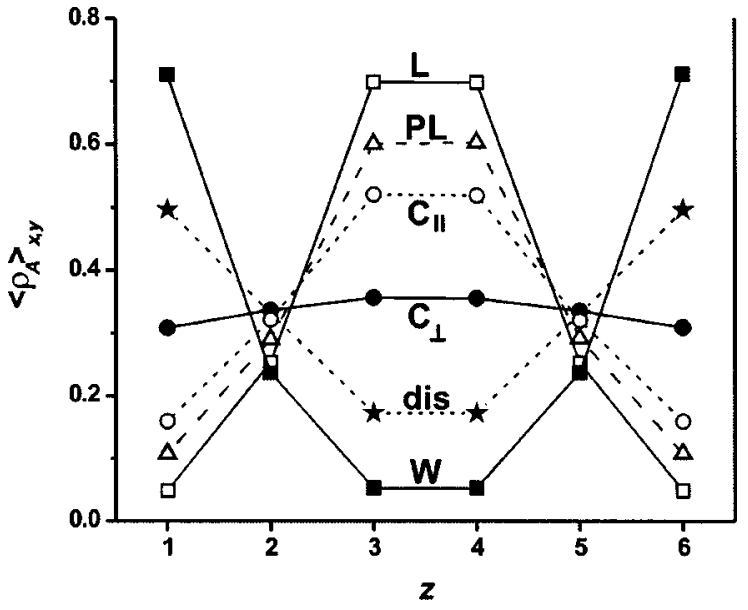

FIG. 7. Effect of the strength of the surface field on the depth profiles of the laterally averaged $A$ density $\left\langle\rho_{A}\right\rangle_{x, y}$ in thin films. Depth profiles are shown for: ( $\square)$ a lamella at $\epsilon_{M}=9,(\triangle)$ a perforated lamella at $\epsilon_{M}=7,(\bigcirc)$ cylinders oriented parallel to the surface at $\epsilon_{M}=5,(\bullet)$ cylinders oriented perpendicular to the surface at $\epsilon_{M}=3,(\star)$ a disordered phase at $\epsilon_{M}=2$, and (घ) a wetting layer at $\epsilon_{M}=-2$.

serve the lamella phase already for $\epsilon_{M}=7$, compared to $\epsilon_{M}$ $=25$ for $H=54$. Also the perforated lamella phase appears already at $\epsilon_{M}=6$ instead of $\epsilon_{M}=10$ and it has a much smaller existence range. Perpendicular cylinders, which at $H=6$ are very short and almost spheres, appear in both cases at $\epsilon_{M}=3$. An additional feature of thin films is the presence of a disordered phase with no well-defined microdomain structure, however, with the two components $A$ and $B$ being still slightly segregated. Figure 7 shows depth profiles of the laterally averaged $A$ density for different structures in thin films of thickness $H=6$. For $\epsilon_{M}<1$, the $A$ block is preferentially attracted to the surface and a wetting layer forms at each surface. For $1 \leqslant \epsilon_{M} \leqslant 2$ the disordered phase forms and the A component is only weakly attracted to the interface. Interfaces with $\epsilon_{M}=3$ appear as neutral. For this surface field value very short cylinders oriented perpendicular to the surface are formed. The fact that the interface appears neutral at $\epsilon_{M}=3$ and not at $\epsilon_{M}=0$ can be explained by an entropic attraction of the shorter $A$ block to the interface. ${ }^{21}$ For $\epsilon_{M}>3$ the surface preferentially attracts the $B$ block and $A$-rich microdomains form in the middle of the film.

\section{Phase diagrams of surface reconstructions}

We have done simulations with $\epsilon_{A B}=6.3,6.5$, and 7.1 and have varied the strength of the surface field, $\epsilon_{M}$, and the film thickness, $H$. We have also calculated a phase diagram where we varied $\epsilon_{A B}$ and $\epsilon_{M}$ simultaneously while keeping $\epsilon_{A B}=\epsilon_{M}$. Figures 8 and 9 show the phase diagrams of surface reconstructions for $\epsilon_{A B}=6.5$ and 6.3, respectively. For both values cylinders are formed in the bulk (see Fig. 2) as well as in the middle of the films. Both phase diagrams clearly show that microdomain structures oriented parallel to the surface are dominant. Cylinders orient perpendicular to the surface for the neutral surfaces at $\epsilon_{M} \approx 3$ and at certain thicknesses $(H=9$ and 15$)$ which strongly deviate from an integer multiple of a natural layer thickness. For hexagonally packed cylinders the natural thickness is $c_{0}=a_{0} \sqrt{3} / 2$, where

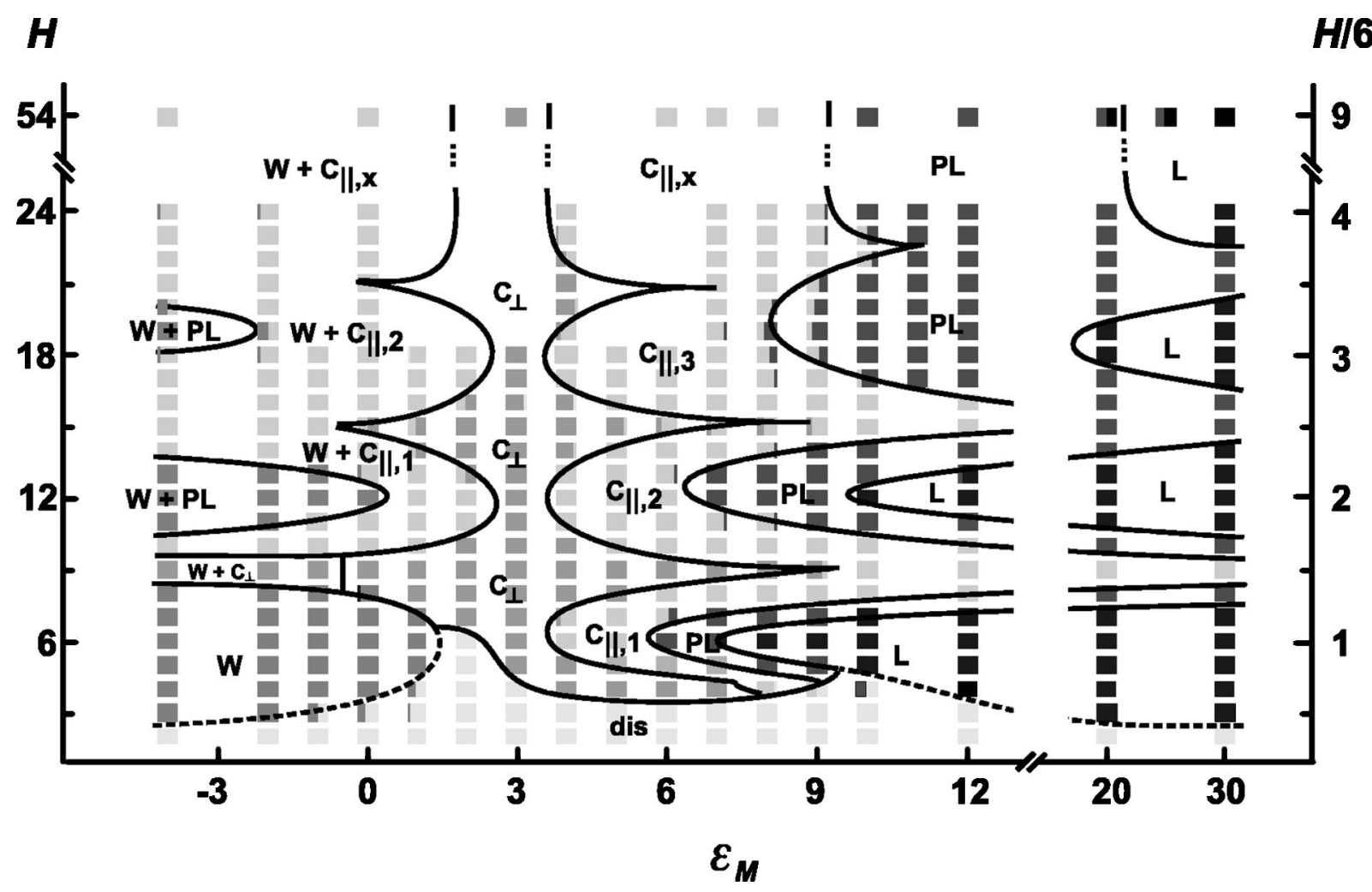

FIG. 8. Phase diagram of surface reconstructions calculated for an $A_{3} B_{12} A_{3}$ melt with $\epsilon_{A B}=6.5$ as function of film thickness $H$ and surface field $\epsilon_{M}$. Boxes indicate where simulations have been done. Boxes with two shades of gray indicate that two phases coexist after the finite simulation time. Smooth phase boundaries have been drawn to guide the eyes. The right scale indicates the film thickness in units of the natural layer thickness $c_{0} \approx 6$. 


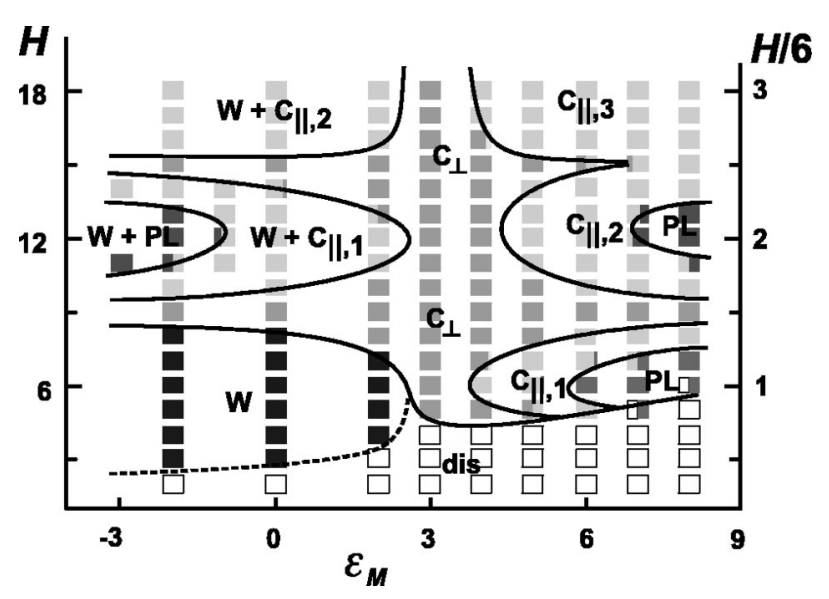

FIG. 9. Same as Fig. 7 but for $\epsilon_{A B}=6.3$.

$a_{0}$ is the distance between next-nearest cylinders in the bulk. In our case (see Sec. I A), $a_{0}=6.9 \pm 0.5$ and the natural thickness of one layer of cylinders is $c_{0} \approx 6$.

Interference of surface fields. The important feature of the thin film phase behavior is the existence of surface reconstructions with noncylindrical morphologies: the wetting layer, the perforated lamella, and the lamella. For thick films with $H>3 c_{0}$ the critical surface field required to induce a surface reconstruction is independent of the thicknesses. For thinner films, this threshold value decreases: for the perforated lamella, $\epsilon_{M} \approx 10,8,7$, and 6 at $H=9 c_{0}, 3 c_{0}, 2 c_{0}$, and $c_{0}$, respectively. This indicates that surface fields extend into the bulk with a decay length of about one microdomain spacing. Furthermore, they are additive and for very thin films the effects of both surfaces combine. This explains why in thin films a weaker surface field is sufficient to form a PL (or L) than in thick films. It also explains the formation of a PL beneath a wetting layer for $\epsilon_{M}=0$ at $H=12$ and $\epsilon_{M}=-2$ at $H=19$.

Confinement effects modulate the stability regions of phase oriented parallel to the interfaces. An integer multiple of a natural layer thickness is energetically favored. This causes easier deformable phases to occur at intermediate film thicknesses. For very small thicknesses $\left(H<c_{0}\right)$ and weak surface fields, confinement prevents microphase separation and stabilizes a disordered phase.

The phase diagram for $\epsilon_{A B}=6.3$ (Fig. 9) displays a very similar behavior to the one for $\epsilon_{A B}=6.5$ (Fig. 8). The two main differences between the two phase diagrams is that for $\epsilon_{A B}=6.3$ the stability region of the disordered phase is larger and that the threshold values for the formation of surface reconstruction are shifted to larger strengths of the surface field, in particular for the lamella surface reconstruction.

Order of phase transitions. An important feature of our simulations is the coexistence of different phases in one layer. Figure 10 shows such a situation where parallel cylinders and a perforated lamella coexist. This simulation was done until 11000 time steps and after 5000 time steps no significant changes were observed. The coexistence of phases corresponds nicely to the experimental observation [see Fig. 1(b) and Refs. 27 and 30]. The presence of coexistent phase clearly indicates a first-order phase transition.

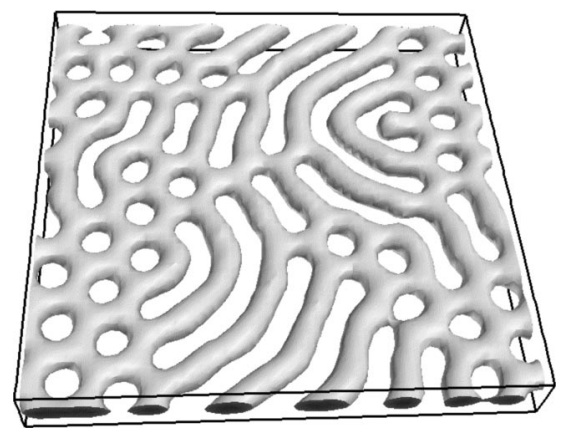

FIG. 10. Coexistence of parallel cylinders and perforated lamellae for an $A_{3} B_{12} A_{3}$ melt with $\epsilon_{A B}=6.5, \epsilon_{M}=6.0$, and $H=7$. The isodensity level is $\rho_{A}=0.45$. The size of the simulation box is $64 \times 64 \times 8$.

The same is also valid for the PL to L transition. The dashed lines in the phase diagrams denote continuous transitions between the W and "dis," and the "dis" and L phase.

\section{E. Structured wetting layer}

A result not displayed in the phase diagrams is the structure of the wetting layer. For thin films $(4 \leqslant H \leqslant 8)$, where the entire film consists only of two wetting layers, the wetting layer has no lateral structure. However, in thicker films and for small values of the surface field $\left(-1 \lesssim \epsilon_{M} \lesssim 2\right)$ the wetting layer has a structure which is complementary to the microdomain structure next to it in the middle of the film (Fig. 11). The entire structure shown in Fig. 11(a) is very similar to hexagonally packed cylinders. ${ }^{37}$ In Fig. 12, histograms of the lateral density distributions within the wetting layer are shown for different values of the surface field $\epsilon_{M}$. For $\epsilon_{M}=1$ and 2, two peaks appear in the histogram which correspond to a lateral microphase separation, for example, stripes of $A$ and $B$ density. For smaller values $\epsilon_{M}$, the two peak merge, which reflects the fact that the structure continuously transforms to a homogeneous wetting layer. Its histogram is similar to that in the middle of a lamella at $\epsilon_{M}$ $=25$ [see Fig. 5(b)] which supports an interpretation as a half lamella.

\section{DISCUSSION}

\section{A. Mapping to the experimental phase diagram}

Our simulations reproduce all essential features of the experimentally observed phase behavior of thin films of polystyrene-block-butadiene-block-polystyrene (SBS) tri-
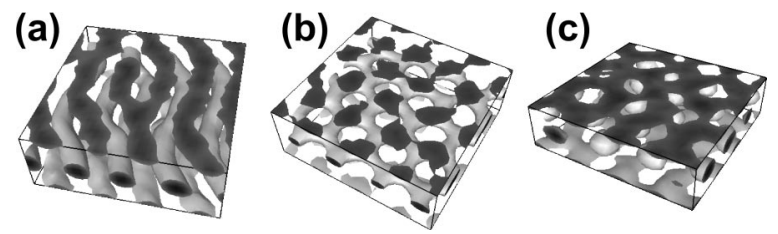

FIG. 11. Structured wetting layers for an $A_{3} B_{12} A_{3}$ melt, $\epsilon_{A B}=6.5$ [simulation box $32 \times 32 \times(H+1)$, after 2000 time steps]. (a) $H=12, \epsilon_{M}=2$, and isodensity level $\rho_{A}=0.45$; the wetting layer resembles half-cylinders. (b) $H=12, \epsilon_{M}=0$, and isodensity level $\rho_{A}=0.45$; the wetting layer consists of isolated dots. (c) A perforated wetting layer at $H=9, \epsilon_{M}=-1$, and isodensity level $\rho_{A}=0.6$. 


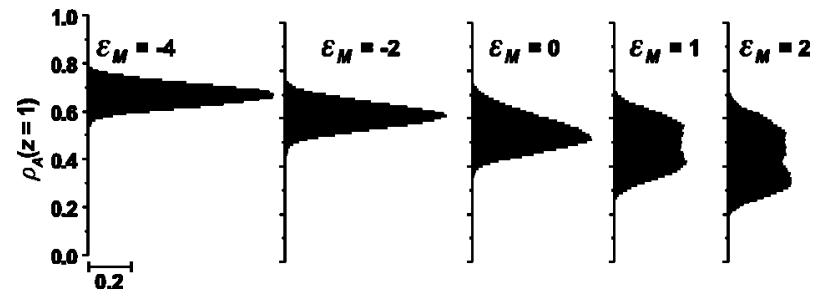

FIG. 12. Effect of the strength of the surface field on the lateral density distribution in the wetting layer. Histograms of the $A$ density at the surface $(z=1)$ are shown for different surface interactions $\epsilon_{M}$. Simulations have been done in $32 \times 32 \times 13$ large simulation boxes with $H=12$. With decreasing $\epsilon_{M}$, the $A$ blocks are more strongly attracted to the surface and the lateral homogeneity of the wetting layer increases.

block copolymers studied by Knoll et al. ${ }^{27,30}$ In particular, the sequence of phases as function of film thickness is correctly modeled. This is nicely seen in Fig. 1(d) where a simulation done in a wedge-shaped geometry is shown. Also the phase diagrams shown in Figs. 8 and 9 nicely match the experimental one (see Fig. 3 in Ref. 27), indicating that the experimental control parameter, the polymer concentration $\Phi_{P}$, is directly related to the control parameter in the simulations, namely the surface field $\epsilon_{M}$.

In order to keep the model as simple as possible we chose to model the SBS/chloroform solution as a melt of $A_{3} B_{12} A_{3}$ block copolymer. As chloroform is a nonselective solvent it acts as a plasticizer, which merely induces chain mobility. ${ }^{27,30}$ The nonselective solvent chloroform screens the interaction between the block copolymer components and the interfaces. This effect is modeled by interaction parameters $\epsilon_{A B}$ and $\epsilon_{M}$, which depend on the polymer concentration $\Phi_{P}$.

The experimentally observed phase diagram (see Fig. 2 in Ref. 27) has three characteristic features: (1) The disordered phase neighbors the $\mathrm{C}_{\perp}$ phase for all polymer concentrations. (2) Both regions of the PL phase have a limited range of polymer concentrations where they are stable. (3) The thicker the film, the higher the critical polymer concentration where the PL appears.

We investigate the range of parameters covered by our simulations [Fig. 13(a)] which give these three characteristic features. As a first reference point, the phases neighboring the disordered phase are shown in Fig. 13(d). The critical phase boundary $\mathrm{C}_{\perp} / \mathrm{C}_{\|, 1}$, which limits the regime where simulations and experimental results are compatible, is shown as a bold dashed line. Figures 13(c) and 13(b) show the phases occurring for $H=c_{0}$ and $2 c_{0}$ including the characteristic phase boundaries $\mathrm{C}_{\|, 1} / \mathrm{PL}$ and $\mathrm{C}_{\|, 2} / \mathrm{PL}$, respectively.

We look for paths in the parameter space which include all three characteristic features. This can be done by projecting the surfaces shown in Figs. 13(b), 13(c), and 13(d) on each other, which is done in Fig. 13(e). The paths have to fulfill the following three conditions: (1) They should completely lay in the $C_{\perp}$ region and should not cross the $C_{\perp} / C_{\|, 1}$ boundary. (2) They should first cross the $\mathrm{C}_{\|, 1} / \mathrm{PL}$ and (3) then the $\mathrm{C}_{\|, 2} / \mathrm{PL}$ boundary.

The gray region displayed in Fig. 13(e) centers at $\epsilon_{M}$ $=6.0$ and corresponds to a region in the experimental phase (a)

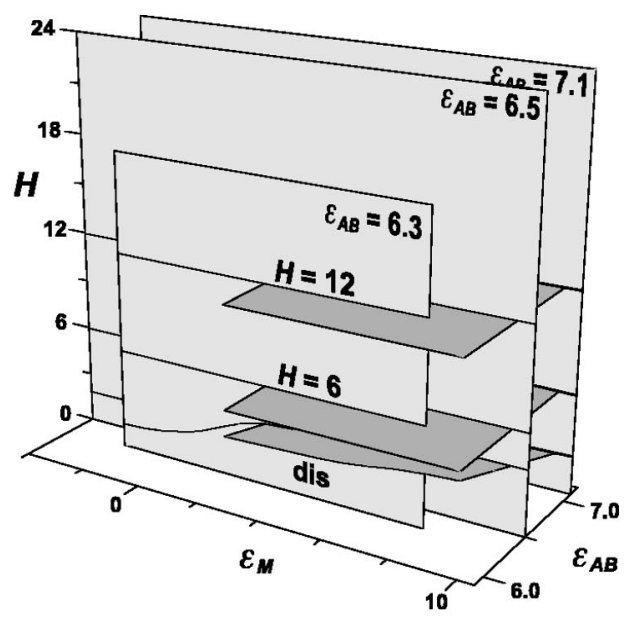

(b)

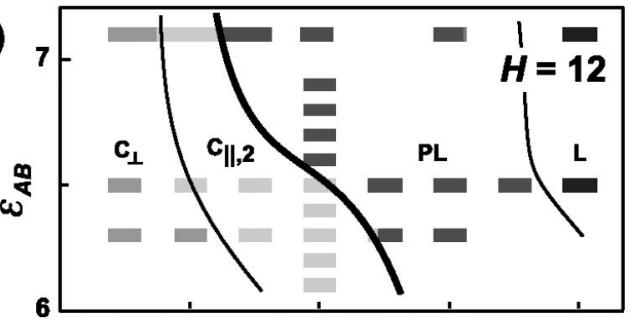

(c)

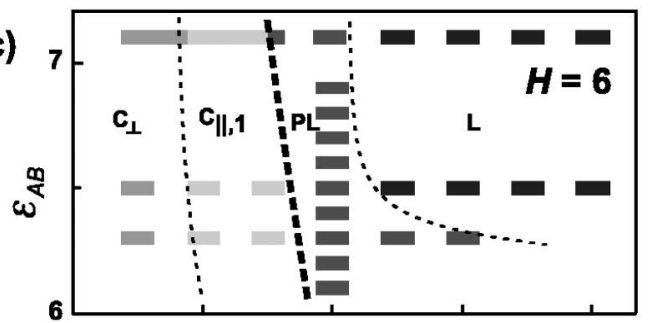

(d)

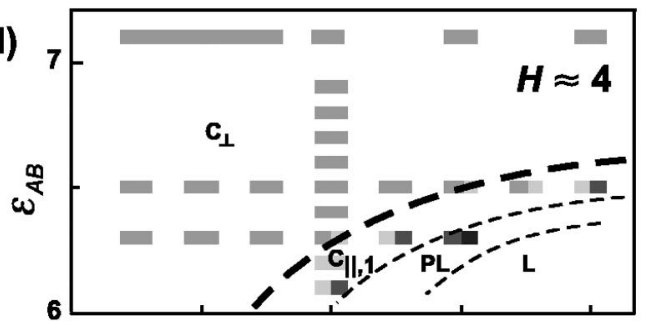

(e)

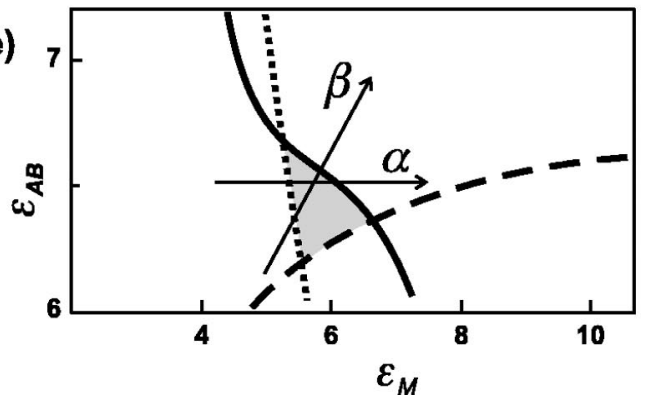

FIG. 13. (a) Range of parameters covered by our simulations. The planes $\epsilon_{A B}=6.3$ and 6.5 correspond to Figs. 8 and 9, respectively. The dark gray surfaces are displayed in detail in (b)-(d). (b) Surface reconstructions formed in films with $H=12$ as function of $\epsilon_{A B}$ and $\epsilon_{M}$. (c) Same as (b) for $H=6$. (d) Surface reconstructions next to the region of the disordered phase. This region is approximatively bounded by $H=4$. Lines indicate phase boundaries. (e) The phase boundaries $\mathrm{C}_{\|, 2} / \mathrm{PL}, \mathrm{C}_{\|, 1} / \mathrm{PL}$, and $\mathrm{C}_{\perp} / \mathrm{C}_{\|, 1}$ taken from (b), (c), and (d), respectively. The arrows $\alpha$ and $\beta$ correspond to two possible models of how the interaction parameters can change with changing polymer concentration $\Phi_{P}$. Both models cross the gray region where a characteristic sequence of phases observed in experiments and simulations coincides. 
diagram centered at $\Phi_{P}=0.59 .{ }^{30}$ Therefore, the most simple way to parameterize such a path is given by the linear relation $\epsilon_{M}=\epsilon_{M}^{\text {melt }} \Phi_{P}$, with $\epsilon_{M}^{\text {melt }}=10 \pm 1$, which is displayed in Fig. 13(e) as arrow $\alpha$. The discrepancy with our previous publication $^{27}$ is due to the fact that the experimental phase diagram was presented in units of the chloroform vapor pressure, whereas here we use the measured polymer concentration from Ref. 30. Nevertheless, both values are close and the physical picture remains the same. By adjusting a single parameter the measured and calculated phase diagrams can be perfectly matched. In particular the predicted $\epsilon_{M}$ values for the onset of the PL phase at $H=6$ and 12 agree nicely with the experiments. Remarkably, the experiments can be described by a parametrization where only $\epsilon_{M}$ changes with $\Phi_{P}$ while $\epsilon_{A B}$ is constant. Other possibilities would be arrows like $\beta$, where both parameters, $\epsilon_{A B}$ and $\epsilon_{M}$, change with $\Phi_{P}$. The choice of the path $\alpha$ is supported by the experimental observation that the SBS/chloroform system studied by Knoll et al. ${ }^{30}$ forms cylinders in the bulk in the whole range of accessed polymer concentrations. This suggests that the influence of $\Phi_{P}$ on $\epsilon_{A B}$ is rather weak. This is consistent with the fact that the gray region in Fig. 13(e) has a considerable larger extent along the $\epsilon_{M}$ axis than along the $\epsilon_{A B}$ axis.

\section{B. Effect of the wetting layer}

In Fig. 14 depth profiles of the laterally averaged $A$ density are compared. The profiles of the film forming the lamella and wetting layer surface reconstruction coincide with that of the film forming parallel cylinders when the profile corresponding to the lamella is shifted by $c_{0}$ and that of the wetting layer is shifted by $c_{0} / 2$. This indicates that the wetting layer can be regarded as a half lamella with thickness $c_{0} / 2$. Furthermore, both the lamella and the wetting layer screen the surface field and the depth profile below them is that of a film forming cylinders oriented parallel to the interface. Effectively, the $A$-wetting layer induces a $B$-rich layer at $c_{0} / 2$, which corresponds to a situation at the interface of a

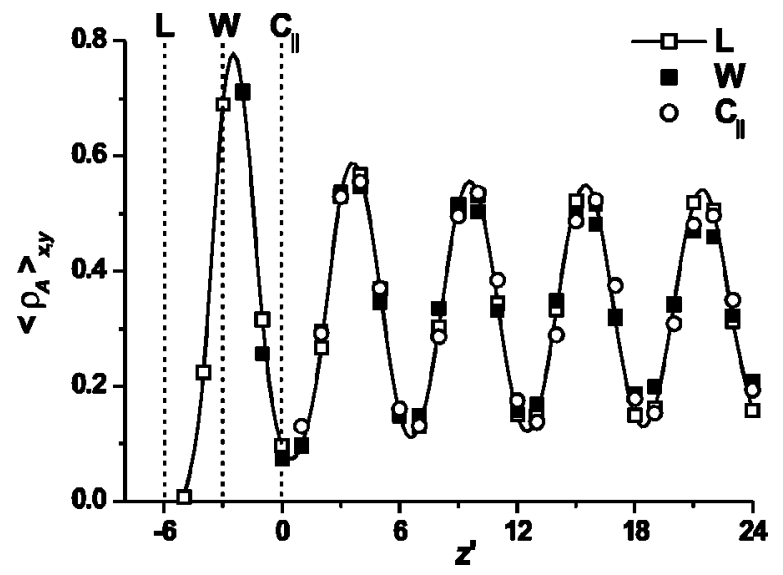

FIG. 14. Depth profiles of the laterally averaged $A$ density $\left\langle\rho_{A}\right\rangle_{x, y}$ in thin films with $H=54$ and $\epsilon_{A B}=6.5$ for different surface fields. The depth profiles are shifted according to $z^{\prime}=z$ for $\mathrm{C}_{\|}\left(\epsilon_{M}=6\right), z^{\prime}=z-3$ for $\mathrm{W}\left(\epsilon_{M}\right.$ $=-4)$, and $z^{\prime}=z-6$ for $\mathrm{L}\left(\epsilon_{M}=30\right)$. The solid line is a spline through the $\mathrm{L}$ data. film which preferentially attracts the $B$ component. The lamella screens a strong surface field in a similar way.

In experiments with supported films the interactions at the air/film and the film/substrate interface are in general different. In a situation where one interface attracts the $A$ and the other the $B$ component, the formation of a wetting layer at one interface can lead to a situation where the film can be treated as having effectively both interfaces attracting the $B$ component. Therefore, the phase diagram measured by Knoll et $a .^{30}$ can be well described in simulations with equal interfaces, although the experiments clearly indicate the presence of an $A$-wetting layer at the film/substrate interface and the preferential attraction of the $B$ component at the air/film interface.

\section{Comparison with cylinder forming diblock copolymers}

The influence of the molecular architecture on the observered phenomena can be studied by comparing our results on $A_{3} B_{12} A_{3}$ triblock copolymers with the behavior of the corresponding $A_{3} B_{6}$ diblock copolymer studied by Huinink et $a .^{21}$ The comparision is made easy since in both studies the same parameters were used and we varied (in addition) the interaction parameter $\epsilon_{A B}$ only slightly. For both systems we are well in the part of the phase diagram where cylinders form in the bulk.

At first glance, no utterly significant difference between the phase diagrams of the $A_{3} B_{6}$ diblock copolymer (Fig. 5 in Ref. 21 and Fig. 4 in Ref. 22) and our $A_{3} B_{12} A_{3}$ triblock copolymers is visible. Only the position of phase boundaries between different phases differs slightly. This fact leads us to the important conclusion that the observed phenomena and mechanisms are present in many cylinder-forming block copolymers. In particular, the molecular architecture plays only a minor role and enters only via the specific values of the interaction parameters. This is further corroborated by results of Wang et al. ${ }^{24}$ obtained with Monte Carlo simulations, which also show a similar phase behavior for cylinderforming systems in thin films.

\section{Comparison with lamella-forming diblock copolymers}

We note that the orientation behavior of the cylinders is analogous to the phase behavior of lamella-forming diblock copolymers as both arc controlled by the interplay between the surface field and confinement effects. ${ }^{4,5}$ Thus, the sequence $\mathrm{C}_{\|} \rightarrow \mathrm{C}_{\perp} \rightarrow \mathrm{C}_{\|}$at steps between terraces corresponds to the sequence $\mathrm{L}_{\|} \rightarrow \mathrm{L}_{\perp} \rightarrow \mathrm{L}_{\|} \cdot{ }^{38}$ Second, in cases where the two confining surfaces favor different orientations $\left(\mathrm{L}_{\|}, \mathrm{L}_{\perp}\right)$ the two orientations can coexist and a hybrid (or mixed) structure (HY) forms ${ }^{39}$ which is similar to cylinders with necks. ${ }^{16}$ We note that in such a HY structure the bulk microdomain structure is preserved and a grain boundary is stabilized in the thin film by the antisymmetric surface field. Furthermore, a disordered phase has been reported for ultrathin films of lamella-forming diblock copolymers ${ }^{3,10}$ and is in nice agreement with our findings and the experiments of Knoll et al. ${ }^{27,30}$ In addition to the alignment effect, hexago- 
nally ordered cylinders can adopt to the planar surface by formation of surface reconstructions (W, PL, L) which also dominate the phase behavior in thin films.

\section{CONCLUSIONS}

Though based on a rather simple microscopic model, our DDFT simulations correctly predict a phase diagram with intriguing complexity. This close match with the experimental data together with the large range of parameters covered by both experiments and simulations, make us believe that we have identified the relevant physical parameters and the mechanisms governing structure formation in the films cylinder forming block copolymers. In particular, the large parameter space covered allows us to distinguish the effects of the two constraints being simultaneously present in a thin film situation: the surface field and the film thickness. Our results also reveal the mechanism of how both interplay.

We identified the deviations from the bulk structure, both in the vicinity of surfaces and in thin films of cylinderforming block copolymers as surface reconstructions. Together with what is known for lamella-forming systems our results give evidence for a general mechanism governing the phase behavior in thin films of modulated phases: The strength of the surface field and the deformability of the bulk structure determines how the system rearranges in the vicinity of the surface. This causes either an orientation of the bulk structure or the formation of surface reconstructions. The stability regions of the different phases are modulated by the film thickness via interference and confinement effects.

This concept along with the presented method might provide the means to understand and eventually control a wealth of thin film structures in a wide class of ordered fluids, such as linear and star multiblock copolymers as well as surfactant based fluids.

\section{ACKNOWLEDGMENTS}

We thank G. Krausch, A. Knoll, and M. Hund for discussions and help. We acknowledge support from the Deutsche Forschungsgemeinschaft (SFB 481), the VolkwagenStiftung, and the NWO-DFG bilateral program.

${ }^{1}$ I. W. Hamley, The Physics of Block Copolymers (Oxford University Press, Oxford, 1998).

${ }^{2}$ F. S. Bates and G. H. Fredricson, Annu. Rev. Phys. Chem. 41, 525 (1990).

${ }^{3}$ S. H. Anastasiadis, T. P. Russell, S. K. Satija, and C. F. Majkrzak, Phys. Rev. Lett. 62, 1852 (1989).

${ }^{4}$ D. G. Walton, G. J. Kellogg, A. M. Mayes, P. Lambooy, and T. P. Russell, Macromolecules 27, 6225 (1994).
${ }^{5}$ G. J. Kellogg, D. G. Walton, A. M. Mayes, P. Lambooy, T. P. Russell, P. D. Gallagher, and S. K. Satija, Phys. Rev. Lett. 76, 2503 (1996).

${ }^{6}$ N. Rehse, A. Knoll, M. Konrad, R. Magerle, and G. Krausch, Phys. Rev. Lett. 87, 035505 (2001)

${ }^{7}$ M. W. Matsen, Curr. Opin. Colloid Interface Sci. 3, 40 (1998).

${ }^{8}$ K. Binder, Adv. Polym. Sci. 138, 1 (1999).

${ }^{9}$ M. J. Fasolka and A. M. Mayes, Annu. Rev. Mater. Sci. 31, 323 (2001).

${ }^{10}$ C. S. Henkee, E. L. Thomas, and L. J. Fetters, J. Mater. Sci. 23, 1685 (1988).

${ }^{11}$ M. A. van Dijk and R. van den Berg, Macromolecules 28, 6773 (1995).

${ }^{12}$ P. Mansky, P. Chaikin, and E. L. Thomas, J. Mater. Sci. 30, 1987 (1995).

${ }^{13}$ G. Kim and M. Libera, Macromolecules 31, 2569 (1998); 31, 2670 (1998).

${ }^{14}$ A. Karim, N. Singh, M. Sikka, F. S. Bates, W. D. Dozier, and G. P. Felcher, J. Chem. Phys. 100, 1620 (1994).

${ }^{15}$ L. H. Radzilowski, B. L. Carvalho, and E. L. Thomas, J. Polym. Sci., Part B: Polym. Phys. 34, 3081 (1996)

${ }^{16}$ M. Konrad, A. Knoll, G. Krausch, and R. Magerle, Macromolecules 33, 5518 (2000).

${ }^{17}$ Ch. Harrison, M. Park, P. M. Chaikin, R. A. Register, D. H. Adamson, and N. Yao, Macromolecules 31, 2185 (1998); Ch. Harrison, M. Park, P. M. Chaikin, R. A. Register, N. Yao, and D. H. Adamson, Polymer 39, 2733 (1998).

${ }^{18}$ Q. Zhang, O. K. C. Tsui, B. Du, F. Zhang, T. Tang, and T. He, Macromolecules 33, 9561 (2000).

${ }^{19}$ S. Turner, M. Rubinstein, and C. M. Marques, Macromolecules 27, 4986 (1994).

${ }^{20}$ G. Brown and A. Chakrabarti, J. Chem. Phys. 101, 3310 (1994); 102, 1440 (1995).

${ }^{21}$ H. P. Huinink, J. C. M. Brokken-Zijp, M. A. van Dijk, and G. J. A. Sevink, J. Chem. Phys. 112, 2452 (2000).

${ }^{22}$ H. P. Huinink, M. A. van Dijk, J. C. M. Brokken-Zijp, and G. J. A. Sevink, Macromolecules 34, 5325 (2001).

${ }^{23}$ G. G. Pereira, Phys. Rev. E 63, 061809 (2001).

${ }^{24}$ Q. Wang, P. F. Nealy, and J. J. de Pablo, Macromolecules 34, 3458 (2001).

${ }^{25}$ J. Feng, H. Liu, and Y. Hu, Macromol. Theory Simul. 11, 556 (2002); J. Feng and E. Ruckenstein, Polymer 43, 5775 (2002).

${ }^{26}$ G. Szamel and M. Müller, J. Chem. Phys. 118, 905 (2003).

${ }^{27}$ A. Knoll, A. Horvat, K. S. Lyakhova, G. Krausch, G. J. A. Sevink, A. V. Zvelindovsky, and R. Magerle, Phys. Rev. Lett. 89, 035501 (2002).

${ }^{28}$ R. J. Spontak, M. C. Williams, and D. A. Agard, Polymer 29, 387 (1988).

${ }^{29}$ R. Magerle, Phys. Rev. Lett. 85, 2749 (2000).

${ }^{30}$ A. Knoll, R. Magerle, and G. Krausch, J. Chem. Phys. 120, 1105 (2004).

${ }^{31}$ J. G. E. M. Fraaije, J. Chem. Phys. 99, 9202 (1993).

${ }^{32}$ J. G. E. M. Fraaije, B. A. C. van Vlimmeren, N. M. Maurits, M. Postma, O. A. Evers, C. Hoffmann, P. Altevogt, and G. Goldbeck-Wood, J. Chem. Phys. 106, 4260 (1997).

${ }^{33}$ G. J. A. Sevink, A. V. Zvelindovsky, B. A. C. van Vlimmeren, N. M. Maurits, and J. G. E. M. Fraaije, J. Chem. Phys. 110, 2250 (1999).

${ }^{34}$ Available from Accelrys Inc., San Diego, CA.

${ }^{35}$ G. H. Fredrickson, V. Ganesan, and F. Drolet, Macromolecules 35, 16 (2002).

${ }^{36}$ M. W. Matsen and R. B. Thompson, J. Chem. Phys. 111, 7139 (1999).

${ }^{37}$ A structured wetting layer similar to the one shown in Fig. 11(a) (halfcylinders) was found theoretically for diblock copolymer thin films by Wang et al. (Ref. 24) and by Pereira (Ref. 23).

${ }^{38}$ B. L. Carvalho and E. L. Thomas, Phys. Rev. Lett. 73, 3321 (1994).

${ }^{39}$ M. J. Fasolka, P. Banerjee, A. M. Mayes, G. Pickett, and A. Balasz, Macromolecules 33, 5702 (2000). 